\title{
Selected Abstracts from the VIIth Biennial Conference of African Society for Immunodeficiencies (ASID): ASID VII Online, Khartoum, October 1-2, 2021
}

\author{
Published online: 11 September 2021 \\ (C) Springer Science+Business Media, LLC, part of Springer Nature 2021
}

Sponsorship: Publication of this supplement was funded by African Society for Immunodeficiencies. All content was reviewed and approved by the Conference Scientific Committee, which held full responsibility for the abstract selections.

Presenting authors have asterisks in the contributor lists.

\section{ABTRACTS FOR ORAL PRESENTATIONS}

\section{S01. Schematic approach to a child with suspected PID}

*Shereen M. Reda ${ }^{1}$

${ }^{1}$ Faculty of Medicine, Ain Shams University, Cairo, Egypt

Background: Inborn errors of immunity (IEI), also known as primary immunodeficiencies (PIDs), represent a heterogeneous group of genetic disorders that affect the development and/or function of cells of the immune system. These disorders manifest with increased susceptibility to infection, autoimmunity, autoinflammatory diseases, allergy, and/or malignancy. Unfortunately, the awareness of PIDs, worldwide, is low among physicians and general practitioners because PIDs are considered relatively rare and complex diseases. Consequently, many patients remain undiagnosed or experience delayed diagnosis leading to increased morbidity and mortality during childhood or later on in adulthood.

Purpose: This oral presentation will demonstrate a systematic approach on when to suspect and how to diagnose PID in infants and children laying stress on symptoms and signs suggestive of these disorders, as well as the basic and advanced laboratory tests that are essential for proper diagnosis. Also, the importance of timely referral of suspected PID cases to specialized centers to confirm diagnosis by other more advanced laboratory and genetic tests and design the proper management plan will be highlighted.

Keywords: Primary immunodeficiencies, Inborn errors of immunity, Approach to diagnosis, Infants, Children.

Disclosure: I have no financial disclosure or conflict of interest with the presented material in this presentation

Corresponding author: Shereen M. Reda shereenreda@med.asu.edu.eg

\section{S02. PID in neonates: Early Diagnosis and Management}

\author{
*Nermeen M.Galal ${ }^{1}$ \\ ${ }^{1}$ Faculty of Medicine, Cairo University, Egypt
}

Background: Primary Immunodeficiency Disorders (PIDs) have different presentations, most of which take time to develop. Newborns, especially preterms, have underdeveloped immune systems which sometimes makes it difficult to identify PIDs easily in these patients.

Purpose: To identify the common presentations of various PID disorders in the neonatal period along with diagnostic pathways and management planning options.

Results: Severe infections, persistent diarrhea, delayed stump separation and or extensive skin lesions may be the initial presentation of PID. Adverse vaccine reactions, autoimmune or autoinflammatory features and or persistent alteration of white cell counts may also point in the direction of PID. Family history of PID or previous sibling death is a major warning sign that calls for PID screening.

Refined diagnosis is not easily accessible in Africa nor is definitive treatment for all cases yet provisional tests and treatment options are discussed.

Conclusion: Early identification via screening / targeted screening in resource limited settings provides excellent results in early detection and prompts initiation of management, if available. This necessitates increasing physicians and public awareness about PID signs in the neonatal period.

Keywords: Primary Immunodeficiency Disorders, early PIDs diagnosis, underdeveloped immune systems, newborn screening.

Disclosures: Author has nothing to disclose/ no conflict of interests.

S03. Genetic diagnostic opportunities for PID: South African Experience

Clair Engelbrecht ${ }^{1}$, Michael Urban ${ }^{1}$, Mardelle Schoeman ${ }^{1}$, Brandon Paarwater $^{1}$, Ansia van Coller ${ }^{2}$, Deepthi Raju Abraham ${ }^{3}$, Helena Cornelissen $^{4}$, Richard Glashoff ${ }^{2}$, Monika Esser ${ }^{2,3}$, Marlo Möller ${ }^{1}$, Craig Kinnear ${ }^{1,5}$, Brigitte Glanzmann ${ }^{* 1}$

${ }^{1}$ SAMRC Centre for Tuberculosis Research, DSI-NRF Centre of Excellence for Biomedical Tuberculosis Research, Division of Molecular Biology and Human Genetics, Faculty of Medicine and Health Sciences, Stellenbosch University, Cape Town, South Africa; ${ }^{2}$ Immunology Unit, Division of Medical Microbiology, National Health Laboratory Service and Faculty of Medicine and Health Sciences, Stellenbosch University, Tygerberg Hospital; ${ }^{3}$ Department of Paediatrics and Child Health, Faculty of Medicine and Health Sciences, Stellenbosch University, Tygerberg Hospital, Cape Town, South Africa; ${ }^{4}$ Division of Hematopathology, 
Tygerberg Hospital, Cape Town, South Africa, National Health Laboratory Service and Faculty of Medicine and Health Sciences, Stellenbosch University, Tygerberg Hospital, Cape Town, South Africa; ${ }^{5}$ SAMRC Genomics Centre, Cape Town, South Africa.

Primary immunodeficiency disorders (PIDs) are inborn errors of immunity (IEI) that cause immune system impairment. To date, more than 400 singlegene IEI have been well defined. The advent of next generation sequencing (NGS) technologies has improved clinical diagnosis and allowed for discovery of novel genes and variants associated with IEI. Molecular diagnosis provides clear clinical benefits for patients by altering management, enabling access to certain treatments and facilitates genetic counselling. Until recently, South African researchers were unable to access affordable platforms capable of human whole genome sequencing locally and DNA samples had to be exported. NGS techniques such as targeted panel screening and whole exome sequencing had to be used on patients with a suspected IEI, with a molecular diagnosis obtained in $30 \%$ of patients. Results highlight the clinical value of expanded genetic testing in IEI and its relevance to understanding the genetic and clinical spectrum of the IEIrelated disorders in Africa. However, detection rates under $40 \%$ illustrate the complexity and heterogeneity of these disorders, especially in an African population, thus highlighting the need for expanded genomic testing such as whole genome sequencing, to identify plausible diseaseassociated variants in these patients.

Corresponding author: Brigitte Glanzmann blindycycle@sun.ac.za

Keywords: inborn errors of immunity, South Africa, whole exome sequencing, targeted sequencing ${ }_{4}$, genetic variants.

Disclosures: Conflicts of interest: None to declare

Funding: Research reported in this publication was partially funded by the National Health Laboratory Service Research Trust (GRANT004 94525), the South African Medical Research Council and the Crick African Network, which receives its funding from the UK's Global Challenges Research Fund (MR/P028071/1). The content is the sole responsibility of the authors and does not necessarily represent the official views of the South African Medical Research Council. This work was also supported by the National Research Foundation of South Africa unique grant number 93360 titled "Identifying novel TB susceptibility candidate genes using NGS data from individuals with PIDs". The funders had no role in study design, data collection and analysis, decision to publish, or preparation of the manuscript.

S04. Mendelian Susceptibility to Mycobacterial Disease in Tuberculosis Hyperendemic South Africa

*Helena Cornelissen ${ }^{1}$, Brigitte Glanzmann ${ }^{2}$, Ansia van Coller $^{3}$, Clair Engelbrecht ${ }^{2}$, Deepthi Raju Abraham ${ }^{4}$, Kessendri Reddy ${ }^{5}$, Marlo Möller $^{2}$, Craig Kinnear ${ }^{2,6}$, Richard Glashoff ${ }^{3}$, Monika Esser ${ }^{3,4}$

${ }^{1}$ Division of Hematopathology, Tygerberg Hospital, Cape Town, South Africa, National Health Laboratory Service and Faculty of Medicine and Health Sciences, Stellenbosch University, Tygerberg Hospital, Cape Town, South Africa; ${ }^{2}$ DSI-NRF Centre of Excellence for Biomedical Tuberculosis Research, SAMRC Centre for Tuberculosis Research, Division of Molecular Biology and Human Genetics, Faculty of Medicine and Health Sciences, Stellenbosch University, Cape Town, South Africa; ${ }^{3}$ Immunology Unit, Division of Medical Microbiology, National Health Laboratory Service and Faculty of Medicine and Health Sciences, Stellenbosch University, Tygerberg Hospital, Cape Town, South Africa; ${ }^{4}$ Department of Paediatrics and Child Health, Faculty of Medicine and Health Sciences, Stellenbosch University, Tygerberg Hospital, Cape Town, South Africa; ${ }^{5}$ Division of Medical Microbiology, National Health Laboratory Service and Faculty of Medicine and Health Sciences, Stellenbosch University, Tygerberg Hospital, Cape Town, South
Africa and Health Sciences, Stellenbosch University, Tygerberg Hospital, Cape Town, South Africa; ${ }^{6}$ SAMRC Genomics Centre, Cape Town, South Africa

Background: Severe infections, in the absence of secondary immunodeficiency, are suggestive of single gene inborn errors of immunity (IEI)/primary immunodeficiency disorders (PIDD). Selective susceptibility to mycobacterial infection, Mendelian Susceptibility to Mycobacterial Disease (MSMD), is characterized by errors in the Interleukin-12-Interferon gamma (IL-12-IFN- $\gamma$ ) pathway.

Objective: To evaluate whether severe, persistent, unusual or recurrent (SPUR) definitions of tuberculosis (TB) can be applied in the context of MSMD in South Africa.

Study design: Between 2013-2018, patients ranging from 0-15 years, meeting SPUR TB definitions of infection were identified. Secondary causes for immunodeficiency were excluded. Basic investigations, then focused immuno-phenotyping and next generation sequencing (NGS) were performed.

Results: A total of 20 patients with a clinical diagnosis of MSMD were identified. Infection with Mycobacterium tuberculosis complex (MTBC) predominated in 64\%. Infection with Bacillus Calmette-Guérin (BCG) infections or non-tuberculous mycobacteria (NTM) infection in $27 \%$, of which two thirds had a pathogenic MSMD variant. Molecular analysis revealed pathogenic variants in $41 \%$ with SPUR mycobacterial infection.

Conclusion: In the South African paediatric population, SPUR TB infections, particularly BCG/NTM, in the absence of a secondary immunodeficiency, may be suggestive of possible MSMD and require further investigation. Diagnosis is complex and requires a multidisciplinary approach with close collaboration between both clinical and research teams.

Corresponding author: Helena Cornelissen hcornelissen@sun.ac.za

Disclosures: Conflicts of interest: None to declare

Funding:

- Crick African Network which receives its funding from the UK's Global Challenges Research Fund (MR/P028071/1)

- National Health Laboratory Service: (GRANT004 94525)

- National Research Foundation Research and Development Fund: (Unique grant number 93360)

- Department of Science and Innovation- National Research Foundation (DSI-NRF) Centre of Excellence in Biomedical Research (UID is 41744)

- SAMRC Centre for Tuberculosis Research

\section{S05. African IEI guidelines: Challenges and future perspectives}

Monika M Esser ${ }^{1}$, Reem Elfeky ${ }^{2}$, Rayan Goda ${ }^{3}$, Ali Sobh $^{4}$, Nahla HH Erwa $^{3,5}$

${ }^{1}$ Division of Immunology \& Medical Microbiology, Department Pathology, Stellenbosch University, Cape Town, South Africa; ${ }^{2}$ Department of Paediatric Stem Cell Transplantation, University Hospital Bristol, UK; ${ }^{3}$ Immunology and Allergy Unit, Soba University Hospital, Khartoum, Sudan; ${ }^{4}$ Mansoura University Children's Hospital, Faculty of Medicine, Mansoura University, Egypt; ${ }^{5}$ Faculty of Medicine, University of Khartoum, Khartoum, Sudan

Background: IEIs are vastly underdiagnosed in Africa. Established reasons include lack of awareness and specialist expertise and networking, confounding infections, phenotype /genotype heterogeneity, African polymorphism of IEI and limited diagnostic facilities - factors which also lead to lack of data/registries for documenting needs and improving patient care. 
Objective: To identify alerting IEI clinical phenotype in the African context and establish relevant diagnostic IEI criteria, adapted to available diagnostic capacity.

Methods: An inclusive task force with African and international advisory board was convened comprising facilitator, team coordinator, scientific chair and chair of core group. Key ASID members are recruited for reviewing African IEI literature, locally endemic relevant diseases and immunodiagnostic capacity for the guideline draft of a specified IEI entity in alignment with IUIS criteria. A structured document protocol approach is followed for the review process, including the following: criteria for diagnosis, differential diagnoses, clinical features/complications, evaluation at first clinic appointment, baseline investigations and samples, prophylaxis and treatment approach, management of complications, follow-up appointments. These drafts are reviewed by the core group before further review with the advisory board members.

Results: The first IEI entities were selected for prominent and distinctive clinical features and important differential diagnostic in Africa, namely ALPS and infantile colitis. Drafts have been reviewed and are prepared for presentation and publication which will enable use for clinical guidance.

Conclusion: African IEIs require locally adapted recommendations for earliest diagnosis and data collection to ensure prompt diagnosis and treatment and planning for health needs of a neglected patient population. Corresponding author: Monika Esser monika@sun.ac.za

S06. African Society for Immunodeficiency guidelines for the diagnosis and management of Autoimmune Lymphoproliferative Syndrome

*Rayan Goda ${ }^{1}$, Reem Elfeky ${ }^{2}$, Nahla HH Erwa ${ }^{1,3,4}$, Monika M Esser ${ }^{5}$

${ }^{1}$ Immunology and Allergy Unit, Soba University Hospital, Khartoum, Sudan; ${ }^{2}$ Department of Paediatric Stem Cell Transplantation, University Hospital Bristol, UK; ${ }^{3}$ Department of Medical Microbiology, University of Khartoum, Khartoum, Sudan; ${ }^{4}$ Clinical Immunology Specialty Council, Sudan Medical Specialization Board, Khartoum, Sudan; ${ }^{5}$ Division of Immunology \& Medical Microbiology, Department Pathology, Stellenbosch University, Cape Town, South Africa

Background: The African Society for Immunodeficiencies (ASID) looked to develop their own guidelines for the diagnosis and treatment of Inborn Errors of Immunity (IEI) in accordance with the International Union of Immunological Societies (IUIS) phenotypic classification. Autoimmune Lymphoproliferative Syndrome (ALPS) was the first to be studied.

Purpose: To form guidelines for the diagnosis and management of ALPS in Africa given the continent's lack of resources as well as the prevalence endemic infectious diseases.

Methods: Retrospective analysis of review articles, case reports and series of ALPS in low resource countries, including African countries.

Results: Diagnostic and management data on ALPS were detected in 8 publications from Egypt, Tunisia and Morocco. Data were compared with ALPS guidelines available from ESID and NIH and the IUIS phenotypic classification. Thorough guidelines were produced for the diagnosis and management of ALPS that considered lack of resources and the prevalent endemic diseases in Africa. A diagnosis of ALPS is considered in patients with a certain number and combination of clinical and laboratory criteria after the exclusion of endemic diseases. Treatment protocols involve multi-disciplinary team approaches, regular patient evaluation and follow up. Drug therapies include corticosteroids and high dose immunoglobulin infusions. Other immunosuppressants are considered for refractory/ severe disease according to step down algorithms.
Conclusion: These guidelines will help raise awareness about ALPS and IEIs in Africa while promoting continentally relevant diagnostic and treatment criteria and inter-center collaborations.

Keywords: Autoimmune Lymphoproliferative Syndrome - Inborn Errors of Immunity (IEIs) - Low-resource countries - Local endemic diseases

Disclosures: All authors have nothing to declare. No conflicts of interest. Corresponding author: Dr Rayan Goda Email: rayangoda@ gmail.com - ORCID ID: 0000-0003-1451-7214

\section{S07. Diagnosis and management of Immune Dysregulation with Colitis; IBD in Africa}

*Ali Sobh ${ }^{1}$, Reem Elfeky ${ }^{2}$, Nahla HH Erwa ${ }^{3}$, Monika M Esser ${ }^{4}$

${ }^{1}$ Department of Pediatrics, Mansoura University Children's Hospital, Faculty of Medicine, Mansoura University, Egypt; ${ }^{2}$ Department of Paediatric Stem Cell Transplantation, University Hospital Bristol, UK; ${ }^{3}$ Immunology and Allergy Unit, Soba University Hospital \& Faculty of Medicine, University of Khartoum; ${ }^{4}$ Division of Immunology \& Medical Microbiology, Department Pathology, Stellenbosch University, Cape Town, South Africa

Purpose: Part of the efforts of the African Society for Immunodeficiency (ASID) is to establish Africa-relevant guidelines for the management of Inborn Errors of Immunity (IEIs). We aimed at producing evidence-based recommendations to guide African doctors in diagnosing and managing Very Early Onset Inflammatory Bowel Disease (VEO-IBD).

VEO-IBD is distinctly differentiated phenotypically and genetically from older-onset IBD and occurs in children less than 6 years old. Diagnosis of monogenic IBD requires various functional and genetic tests. Most studies suggest that VEO-IBD patients may be more refractory to standard anti-inflammatory and immunomodulatory drugs.

Methods: We searched PubMed for International and African publications about VEO-IBD in patients with primary immunodeficiency.

Results: Very few case reports and case series described this important phenotype in Africa. Globally, there is little data to guide optimal management of VEO-IBD. International efforts are running to better understanding the progression of disease and to ascertain the best therapeutic interventions.

Conclusion: Further research is required for better understanding and tailoring evidence-based guidelines for the diagnosis and management of VEO-IBD. We suggest an African approach for the diagnosis and management for these patients based on available international guidelines and tailored according to available diagnostic and treatment facilities, with emphasis on specialized centers which can help countries with limited resources.

Keywords: very early onset inflammatory bowel diseases (VEO-IBD), Primary immunodeficiency, Inborn errors of immunity (IEI), Colitis, Immune Dysregulation

Disclosures: Nothing to disclose

Corresponding author: Ali Sobh ali.sobh@mans.edu.eg

O01. Utility of expanded molecular testing for primary immunodeficiencies: disease-specific panels may miss diagnoses

*Alekhya Narravula ${ }^{1}$, Mahmoud Husseini ${ }^{1}$, Jessica Connor ${ }^{1}$, RebeccaTruty $^{1}$, Jennifer Holle ${ }^{1}$, Shiloh Martin ${ }^{1}$, Hui Yu ${ }^{1}$, Olga Sarmento ${ }^{1}$, Britt Johnson ${ }^{1}$

${ }^{1}$ Invitae corporation, San Francisco, CA, USA

Purpose: Primary immunodeficiency disorders (PIDs) exhibit "phenotype expansion" in which genes previously thought to be associated with specific phenotypes have an increasing circle of clinical presentations. 
Traditionally, genetic testing for PIDs is phenotype-specific, guided by genes associated with a given clinical presentation. However, the performance of disease specific panels versus broad panel testing has not been performed.

Methods: This study compared the diagnostic value of a 207 gene PID panel to smaller, disease focused panels. Based on patient phenotypic information provided, each requisition was assigned a virtual disease-focused panel. Positive diagnoses on the expanded panel and the virtual focused panel were compared for each requisition to determine the frequency of missed diagnoses if only the focused panel had been ordered.

Results: Our laboratory performed 6,257 expanded PID panels between April 2017 and October 2019. A virtual focused panel could be assigned based on clinical indication for 3,384 unique orders, 105 of which had positive results. Positive results would have been missed in $24.8 \%(26 / 105)$ of cases had the expanded panel not been ordered. The Antibody Deficiencies panel had the highest rate of missed diagnoses $(42.5 \%)$.

Conclusion: These results indicate the importance of ordering an expanded panel or having the option to re-requisition to the expanded panel for conditions with overlapping symptoms.

\section{O02. Computational Analysis Revealed Five Novel Mutations in Human IL2RG gene Related to X-SCID}

*Tamadur Babiker Abbas Mohammed ${ }^{1,2}$, Asma Ali Hassan Ali ${ }^{1,3}$, Areeg Elsir Abdelgadir Elemam ${ }^{1,4}$, Wala Omer Mohammed Altayb ${ }^{1}$, Tebyan Ameer Abdelhameed Abbas ${ }^{1}$, Mohamed Ahmed Salih Hassan ${ }^{1}$

${ }^{1}$ Department of Bioinformatics, Africa City of Technology, Khartoum, Sudan; ${ }^{2}$ Department of Clinical Immunology, Haj AL Safi Teaching Hospital, Khartoum, Sudan; ${ }^{3}$ Faculty of Science, Department of Zoology, University of Khartoum, Khartoum, Sudan; ${ }^{4}$ Department of Physiology, Al-Neelain University, Khartoum, Sudan.

Background: X-linked severe combined immunodeficiency is a rare lifethreatening disorder that presents in early infancy with severe recurrent opportunistic infection.

Purpose: To use bioinformatics tools for identifying effects of possible pathogenic Single Nucleotide Polymorphisms (SNPs) on structure and function of IL2RG gene as a cause of X-linked SCID.

Method: Data on the IL2RG gene was gathered from the dbSNP/NCBI database. Prediction of damaging effect was done using bioinformatics software: SIFT, Polyphen, Provean, SNAP2, SNP\&GO, PHD-SNP.PMut, I-mutant, Project HOPE, PolymiRTS and GeneMAINIA. The effects of the mutations on the 3D structure of the human IL2RG protein were predicted using RaptorX and visualized by Chimera.

Result: In-silico prediction identified 1479 SNPs within the IL2RG gene, 253 out of them presented at the coding region and 50 SNPs took place in the miRNA 3'UTR while 21 occurred in 5'UTR region and 921 occurred in intronic regions. Using bioinformatics tools, 12 nsSNPs damaging IL2RG coding region were found. Five nsSNPs were identified as novel SNPs including: G305R, C182Y, G114D, Y105C and Y91C. Moreover, 3'UTR region analysis showed two SNPs out of 50 (rs144075871 and rs191726889) were predicted to disrupt miRNAs binding sites which affect gene expression.

Conclusions: This study revealed 5 novel nsSNPs in the IL2RG gene by using different software and two SNPs disrupting miRNAs binding sites in 3'UTR region. These SNPs might be considered as an important factor in causing diseases related to IL2RG gene mutation and could be used in the diagnosis of X-linked SCID.

Keywords: X linked severe combined immunodeficiency (X-SCID), interleukin 2 receptor gamma-chain (IL2RG), single nucleotide polymorphism (SNP), nonsynonymous Single Nucleotide Polymorphisms (nsSNPs), bioinformatics

Corresponding author: Abbas B. Tamadur tamadurbabiker@gmail.com
O03. Health-related quality of life of patients and families with primary immunodeficiency in Malaysia: A cross-sectional study

*Ruwaydah Ahmed Meelad ${ }^{1}$, Intan Juliana Abd Hamid ${ }^{1}$, Zarina Thasneem Zainudeen ${ }^{1}$, Ilie Fadzilah Hashim ${ }^{1}$, Muhd Nur Akmal Azizuddin ${ }^{1}$, Ernest Mangantig ${ }^{1}$, Fahisham Taib ${ }^{2}$, Norsarwany Mohamad $^{2}$, Intan Hakimah Ismail ${ }^{3}$, Amir Hamzah Abdul Latiff ${ }^{4}$, Lokman Mohd $\mathrm{Noh}^{5}$

${ }^{1}$ Primary Immunodeficiency Group, Cluster of Regenerative Medicine, Advanced Medical and Dental Institute, Universiti Sains Malaysia, 13200 Kepala Batas, Penang, Malaysia; ${ }^{2}$ Paediatric Department, School of Medical Sciences, Universiti Sains Malaysia, 16150, Kubang Kerian, Kelantan; ${ }^{3}$ Department of Paediatrics, Faculty of Medicine and Health Sciences, Universiti Putra Malaysia, 43400 Serdang, Selangor, Malaysia; ${ }^{4}$ Pantai Hospital, Kuala Lumpur, Malaysia; ${ }^{5}$ Department of Paediatrics, Hospital Tunku Azizah, Ministry of Health Malaysia, Kuala Lumpur, Malaysia.

Purpose: Primary immunodeficiency diseases (PID) affect various aspects of a patient's life. However, the health-related quality of life (HRQOL) of PID patients in Malaysia is poorly described. This study aimed to determine the quality of life of Malaysian PID patients and parents.

Method: This cross-sectional study was performed from August 2020 to November 2020. Patients with PID and their families were invited to answer the PedsQL Malay version (4.0) questionnaire, the tool used to assess the HRQOL. A total of 41 families and 33 patients with PID answered the questionnaire. A comparison was performed with the previously published value of healthy Malaysian children.

Result: Parents of respondents recorded a lower mean of total score than the parents of normal healthy children $(67.26 \pm 16.73$ vs. $79.51 \pm 11.90$, p-value $=$ 0.001 , respectively). PID patients reported lower mean total score to normal healthy children $(73.68 \pm 16.38 \mathrm{vs} .79 .51 \pm 11.90$, p-value $=0.04)$, including psychosocial domain $(71.67 \pm 16.82$ vs. $77.58 \pm 12.63$, p-value $=0.05)$, and school functioning, $(63.94 \pm 20.87$ vs. $80.00 \pm 14.40, p$-value $=0.007)$. No significant difference of reported HRQOL when comparing between subgroup of PID on immunoglobulin replacement therapy and those without immunoglobulin replacement (56.96 \pm 23.58 vs. $65.83 \pm 23.82$, p-value 0.28$)$. Socioeconomic status was found to be predictive of the lower total score of PedsQL in both parent and children reports.

Conclusion: Parents and children with PID, especially those from middle socioeconomic status, have lower HRQOL and school function impairment than healthy children.

Keywords: Health-related quality of life, Inborn errors of immunity, Primary Immunodeficiency, PedsQL, Malaysia

Corresponding author: Intan Juliana Abd Hamid: intanj@usm.my

O04. Inborn Errors of Immunity with Invasive Fungal Infections: A Single Center Experience

*Rasha El-Owaidy, Dalia El-Ghoneimy, Nesrine Radwan, Ghada Shousha, Roba Al-Gaweesh, Rana Zakaria, Mostafa Al-Amin, Elham Hossny, Zeinab A. El-Sayed, Yehia El-Gamal, Shereen M. Reda

Paediatric Allergy and Immunology Unit, Children's Hospital, Ain Shams University, Cairo, Egypt

Purpose: Data about invasive fungal infections in patients with inborn errors of immunity (IEI) in Egypt are still inadequate.

Methods: We herein describe fungal infections encountered in a group of infants and children with IEI.

Results: Out of 680 patients with IEIs, 42 patients had documented invasive fungal infections. They were 26 boys $(62 \%)$ and 16 girls (38\%) with age ranging $0.3-13$ years, median 3.5 years. According to their diagnoses, 15 had chronic granulomatous disease (35.7\%), 6 
severe combined immunodeficiency (14.3\%), 3 LRBA mutation (7\%), 2 CD3 lymphopenia (4.7\%), 2 congenital neutropenia (JAGN1 mutation), one autoimmune lymphoproliferative syndrome and one with each of the following mutations: STAT3 GOF, STAT 1 GOF, CARD 9, CARD 11, TRNT1, DOCK8 and PIK3C2G. The remaining 6 patients are still under investigation. Mucocutaneous infections were the most common $(22,52.4 \%)$ followed by pulmonary affection $(19,45.2 \%)$, bone/joint lesions $(8,19 \%)$, intracranial lesions $(7,16.7 \%)$, hepatic lesions $(4,9.5 \%)$, eye invasion $(3,7 \%)$ and intracardiac fungal masses in $2(4.7 \%)$. Aspergillus and Candida species were equally prevalent among the described cases (each in 21 patients; 50\%), followed by Pneumocystis jirovecii ( $\mathrm{n}=3,7 \%)$, Mucormycosis in one and Exophilia dermatitidis in one. Antifungal therapy included voriconazole in 31 (73.8\%), amphotericin in 24 (57\%), fluconazole and posaconazole, each in 9 (21.4\%), and anidulafungin in $2(4.7 \%)$, while 14 patients $(33.3 \%)$ required combined antifungal therapy. The mortality rate among our patients reached 23/42 (54.8\%).

Conclusion: Timely diagnosis of fungal infections in patients with IEI and antifungal availability are still challenging.

Keywords: Fungal, Aspergillus, Candida, invasive, mortality, pediatric, immunodeficiency

\section{O05. Mendelian Susceptibility to Mycobacterium Disease (MSMD):} A Single Center Experience

*Nesrine Radwan ${ }^{1}$, Rasha El-Owaidy ${ }^{1}$, Dalia H El-Ghoneimy ${ }^{1}$, Ghada Shosha ${ }^{1}$, Hanan Abdel Latif ${ }^{1}$, Amira Fouad ${ }^{1}$, Mariam abel-Naby, Roba Gawish $^{1}$, Rana Zakaria ${ }^{1}$, Mostafa Al-amin ${ }^{1}$, Bothina A H Taha ${ }^{2}$, Eman MI Tantawy ${ }^{2}$, Basma SAM Mansour ${ }^{2}$, Jacinta Bustamante ${ }^{3,4,5,6}$, JeanLaurent Casanova ${ }^{3,4,5,6}$, Elham Hossny ${ }^{1}$, Ashraf Abdel Baky ${ }^{1}$, Gehan Mostafa $^{1}$, Yehia El-Gamal ${ }^{1}$, Shereen M Reda ${ }^{1}$, Zeinab A El-Sayed ${ }^{1}$.

${ }^{1}$ Pediatric Allergy and Immunology Unit, Faculty of Medicine, Ain Shams University, Cairo, Egypt; ${ }^{2}$ Abassyia Chest Hospital, Cairo, Egypt; ${ }^{3}$ University of Paris, Imagine Institute, Paris, France; ${ }^{4}$ Laboratory of Human Genetics of Infectious Diseases, Necker Branch, INSERM U1163, Necker Hospital for Sick Children, Paris, France; ${ }^{5}$ Center for the Study of Primary Immunodeficiencies, Necker Hospital for Sick Children; Paris, France; ${ }^{6}$ St. Giles Laboratory of Human Genetics of Infectious Diseases, Rockefeller Branch, The Rockefeller University, New York, USA.

Background: Mendelian susceptibility to mycobacterium tuberculosis (MSMD) is a rare group of inherited conditions characterized by selective predisposition to mycobacterial infection. We here demonstrate the clinical and molecular data of patients diagnosed with MSMD in our center.

Methods: One hundred infants and children with mycobacterial infection not responding to triple antituberculous therapy were evaluated for possible underlying Inborn errors of Immunity (IEI). Their clinical and immunological data were evaluated, and patients with MSMD apart from chronic granulomatous disease and other IEI were described.

Results: Out of the 100 patients screened, 18 patients were suspected to have MSMD based on their clinical data and normal basic immunological tests. They were 12 females and 6 males (ratio 2:1). Their median age of onset, age at diagnosis and diagnostic lag were $6.5,24$ and 19 months respectively. Seventeen patients received live attenuated BCG vaccine at birth. Ten patients had recurrent fungal infections, four had disseminated salmonella infections and 3 patients had history of encephalitis. Genetic diagnosis was performed to eleven patients and it showed IL12RB1 mutation in all. Subcutaneous interferon gamma was administered to two patients to control infection but for a short course due to unsustained availability and high cost of the drug. All patients were from consanguineous families, mainly from upper Egypt (13/18 patients) and four had positive family history. Three patients died out of fulminant infection soon after diagnosis. None of the patients received hematopoietic stem cell transplantation.

Conclusion: IL12RB1 mutation is elicited as the only mutation in our small cohort; however, more patients need to be screened to confirm this finding and determine other possible mutations causing MSMD in Egypt. Keywords: Mendelian Susceptibility, Tuberculosis, Inborn error of Immunity

O06. Genotype-Phenotype of Mevalonate kinase deficiency: a potentially life threatening disease

*Dalia H. El-Ghoneimy ${ }^{1}$, Rasha El-Owaidy ${ }^{1}$, Nesrine Radwan ${ }^{1}$, Ghada Shousha $^{1}$, Hanan Abdel-Latif ${ }^{1}$, Amira Fouad ${ }^{1}$, Roba Al-Gaweesh ${ }^{1}$, Rana Zakaria $^{1}$, Mostafa Al-Amin ${ }^{1}$, I. Jéru ${ }^{2}$, S. Amselem ${ }^{2}$, Eman D. ElDesouky $^{3}$, Yehia El-Gamal ${ }^{1}$, Zeinab A. El-Sayed ${ }^{1}$, Shereen M. Reda ${ }^{1}$, Elham Hossny ${ }^{1}$.

${ }^{1}$ Pediatric Allergy, Immunology and Rheumatology Unit, Ain Shams University, Cairo, Egypt; ${ }^{2}$ Institut National de la Santé et de la Recherche Médicale (INSERM), Université Pierre et Marie Curie (UPMC), Paris, France; ${ }^{3}$ Epidemiology and Biostatistics, NCI, Cairo University

Background: Mevalonate kinase (MVK) deficiency (formerly known as hyperimmunoglobulin D syndrome) has different clinical presentation of variable severity, response to treatment and outcome. A link has been described between the underlying mutation in MVK gene and the resulting phenotype.

Patients and methods: Six patients presenting with episodes of prolonged fever since early infancy were evaluated for possible MVK deficiency.

Results: Five patients are Egyptians and one patient was Syrian. They were 4 boys $(67 \%)$ and 2 females $(33 \%)$. The age at onset of their presentation ranged between 0.7-4 months with a median (IQR) of 3(1-4) months. Positive parental consanguinity was found in 5 patients $(83 \%)$. All patients have prolonged episodes of high fever $(40$ ${ }^{\circ} \mathrm{C}$ ), generalized lymphadenopathy and hepatosplenomegaly. Nondeforming arthritis was present in 5 patients $(83 \%)$. Abdominal pain and chronic diarrhea were present in 3 patients $(50 \%)$. No neurological deficit nor dysmorphic features were found in any of these patients. All patients had persistent leucocytosis, elevated ESR and CRP. Immunoglobulin D was elevated in one patient $(203 \mathrm{mg} / \mathrm{L})$. The mutation of the MVK gene has been identified in 4 patients as: p.H20Q (c. T60A) in the 2 brothers (one deceased); p.R388X (c.C1162T) in the Syrian girl (deceased); p. G 326 R (c.976G>A) in one patient (alive).Cause of death in the 2 patients was uncontrolled disease activity and macrophage activation syndrome. Anakinra was the best treatment to put patients in complete remission.

Conclusion: MVK deficiency could be a life threatening disease. Availability of genetic diagnosis will help in early diagnosis of suspected cases and support initiation of anakinra in severe cases.

P001. The pharmacology of human polyvalent immunoglobulins in patients with primary immunodeficiency diseases

*Nouhaila Mouadny ${ }^{1}$, Samya Moussamih ${ }^{2}$, Jalila EL Bakkouri ${ }^{1,3}$, Aziz Bousfiha $^{1,4}$

${ }^{1}$ Laboratory of clinical immunology, inflammation and allergy, Faculty of Medicine and Pharmacy of Casablanca, Hassan II University, Morocco; ${ }^{2}$ Laboratory of Immunology and Biodiversity, Department of Biology, Faculty of Sciences Aïn Chock, Hassan II University, Casablanca, Morocco; ${ }^{3}$ Laboratory of Immunology, Ibn Rochd University Hospital, Casablanca, Morocco; ${ }^{4}$ Unit of Clinical Immunology, Department of Infectious Diseases, Hospital A. Harouchi Hospital, Ibn Rochd University Hospital, Casablanca, Morocco. 
Introduction: Primary immunodeficiency diseases (PID) encompass more than 400 molecularly defined disorders that affect the development and/or function of the immune system. Human intravenous immunoglobulin (IVIG) has been used in the treatment of many diseases, and its effects include complex mechanisms. Although the specific indications for IVIG are limited, it has been shown to be useful in many diseases in clinical practice.

In this study, the use of IVIG, its mechanisms of action, indications and side effects were discussed, and then the research question was answered: "Is the therapeutic dose of $0.5 \mathrm{~g} / \mathrm{kg}$ that all PID patients receive sufficient to provide a protective residual dose?"

Methods: This study took place over a period of 3 months in the Infectious Diseases and Clinical Immunology Unit at the IBN ROCHD Children's Hospital in Casablanca. We collected 25 Moroccan children with PID who were treated with IVIG (a monthly dose of $0.5 \mathrm{~g} / \mathrm{kg} / \mathrm{month}$ ). The determination of residual IgG was carried out by the turbidimetry in the Immunology laboratory of University Hospital.

Results: The distribution of patients according to the type of PID revealed that $44 \%$ were cases of Primary Antibody Deficiency (PAD) and 56\% had combined immunodeficiency (CID). The average age of patients was 9 years. The mean residual dose of IgG in children with PAD was $(5.03( \pm \mathbf{2 . 2 6}) \mathrm{g} / \mathrm{l})$, and $(8.575( \pm 6.30) \mathrm{g} / \mathrm{l})$ in patients with CID. We observed adverse events in 5 patients $(20 \%)$ of our population.

Conclusion: The comparison of our results with the literature data, allowed us to answer the research question posed: The therapeutic dose of $0.5 \mathrm{~g} / \mathrm{kg}$ that patients receive every 3 to 4 weeks is sufficient to give a protective residual dose $\geq 5 \mathrm{~g} / \mathrm{l}$, and that the residual IgG level increases significantly with each increase in the infused dose.

Keywords: Primary immune deficiency, IVIG, SCG, IgG residual dose

P002. Expression of High Viral Load among adult Sudanese HIV positive patients as a risk factor for tuberculosis in Omdurman HIV center

*Mohamed Eltayeb ${ }^{1}$

${ }^{1}$ Sudan Medical Specialization Board, Sudan, Khartoum

Background: Tuberculosis (TB) infection is the leading cause of death worldwide from a single infectious agent. TB is also the leading killer of HIV-positive patients.

Purpose: This study aimed at investigating the expression of high viral load among adult Sudanese HIV positive patients, as a risk factor for TB. The data were expected to give an insight into the epidemiology of both HIV and TB infections in Sudan.

Methods: A cross-sectional study which was conducted in Omdurman HIV center during the period between September 2020 and January 2021. Data was extracted from the patients' records. The study population was based on adult patients ( $>16$ years) who were diagnosed and started Anti Retroviral Therapy (ART) programs in 2018 and had viral load records. HIV negative patients, patients without viral load records and patients $<16$ years were excluded. The data were collected by using a questionnaire which included age, gender, TB status (pulmonary and extra-pulmonary), HIV WHO clinical stage, method of TB diagnosis, HIV-RNA levels.

Results: $25 \%$ of 100 patients with HIV had TB. Men were more infected than women and patients $>50$ years of age were the least affected while subjects aging $20-50$ years were the most affected. There was no significant difference in the viral load in patients with HIV/TB compared with patients with HIV alone; $p$ value: 0.35 . The methods of TB diagnosis were correlated with the stage of HIV stage of infection; $p: 0.02$. It was also observed that stage II of HIV infection was predominant.

Conclusion. HIV/TB coinfection is increasing. The most common diagnostic method for TB was microscopy. It is not necessary for patients with HIV/TB to have high viral load.
P003. Features of Immune Dysregulation in Primary Immunodeficiency Patients at Soba University Hospital, Khartoum -Sudan

*Rayan Goda ${ }^{1}$, A.SI. Mohamed ${ }^{1}$, A Salim ${ }^{1}$, Sulum O Masoud ${ }^{1}$, Nahla HH Erwa ${ }^{1,2,3}$

${ }^{1}$ Immunology and Allergy Unit, Soba University Hospital, Khartoum Sudan; ${ }^{2}$ Faculty of Medicine Department of Medical Microbiology, University of Khartoum, Khartoum, Sudan; ${ }^{3}$ Clinical Immunology Specialty Council, Sudan Medical Specialization Board, Khartoum, Sudan

Background: We reviewed all patients with primary Immunodeficiency diseases (PID) that presented to immunology clinic at our hospital for features of immune dysregulation.

Purpose: To describe the clinical manifestations and basic immunological findings in Sudanese patients with features of immune dysregulation. Additionally, we looked into how these findings affected their management and outcome.

Methods: Retrospective analysis of all patients referred with PID looking for features of immune dysregulation: autoimmunity, allergy, lymphoproliferation and cytopenias.

Results: Total number of patients with suspected PID was 120 patients. Sixteen patients had features suggestive of immune dysregulation in the form of autoimmunity evident as autoimmune cytopenia, endocrinopathies, lymphoproliferation, inflammatory bowel disease, enteropathy, arthropathy and allergy. Most reported allergic features included eczema and food allergy. Half of the patients had altered immune phenotypes with low immunoglobulin levels; either $\operatorname{IgG}$ or $\operatorname{IgA}$ ( 5 patients), increased $\operatorname{IgE}$ was noted in 4 patients. Four patients $(25 \%)$ had low CD4 helper T cells, increased doublenegative T (DNT) cells were seen in 4 patients and one patient had low CD4+CD25+FOXP3+ T cells. Management proved difficult due to recurrent infections, medication side effects or refractory disease. Almost half of the patients were lost to follow up, with 3 deceased patients.

Conclusion: Immune dysregulation in patients with PIDs is poorly identified and hence under reported in Sudan and Africa. Inter-clinic collaboration is recommended to identify more patients, with the provision of better diagnostic and therapeutic modalities including targeted therapy and specific genetic diagnosis.

Disclosures: Authors have nothing to disclose and report no conflict of interest.

Keywords: Primary Immunodeficiency Diseases, Immune dysregulation, allergy, autoimmunity, lymphoproliferation.

Corresponding author: Dr Rayan Goda - Email address: rayangoda@gmail.com

P004. Knowledge and Practice of Doctors on Prescribing Intravenous Human Polyclonal Immunoglobulins among Sudanese Doctors

*Sulum O Masoud ${ }^{1,2}$, Hala K Ali ${ }^{2,3}$, Nahla HH Erwa ${ }^{1,2,4}$

${ }^{1}$ Immunology and Allergy Unit, Soba University Hospital, Khartoum, Sudan; ${ }^{2}$ Clinical Immunology Specialty Council, Sudan Medical Specialization Board, Khartoum, Sudan; ${ }^{3}$ Faculty of Medicine, International University of Africa, Khartoum, Sudan; ${ }^{4}$ Faculty of Medicine, Department of Medical Microbiolog, University of Khartoum, Khartoum, Sudan

Introduction: Intravenous immunoglobulins (IVIG) is produced from pooled plasma obtained from thousands of blood donors, hence the potential hazards of viral transmission, adverse reactions, and the growing difficulties with obtaining safe plasma, all of which impact patients'health and products cost. Not much is known about the knowledge and practice of doctors on prescription 
IVIG in Sudan which have important effects on the rational use of the products.

Objectives: To investigate knowledge, and practice of prescribing IVIG with regards to rational use, doses and side effects, among treating doctors in referral hospitals within Sudan. Another objective was to identify the limitations of prescribing IVIG among doctors.

Methodology: A descriptive cross-sectional study using non-supervised questionnaires, designed based on multiple choice, and "self-rating" questions distributed to IVIG prescribers at 7 referral hospitals located in Khartoum, Sudan (April-December 2018).

Results: A satisfactory knowledge score (7-13) was seen in 64\% among participants. Thirty five percent of physicians, mainly junior doctors reflected an unsatisfactory level of knowledge (0-6), while $1 \%$ scored high rates (14-20). A satisfactory practice score (7-13) was seen in 54\% of participants in contrast to unsatisfactory (0-6) in 43\%. Products cost, shortages, and lack of educational programs were the main limitations to using IVIG among Sudanese doctors.

Conclusions: These results call for implementation of training programs for doctors who are prescribing IVIG. Continuous educational and training programs must be conducted for physicians to ensure rational use of the products.

Keywords: IVIG, study, Intravenous Immunoglobulin G, IVIG indication.

P005. Recurrent anaphylaxis in an infant with Cutaneous Mastocytosis

*Ghada A Shousha

Department of Pediatrics, School of Medicine, Ain Shams University, Cairo, Egypt

Purpose: Rashes during infancy are mostly secondary to infection or allergy but can be due to rare serious disorders with poor prognosis; hence, the importance of clinical differential diagnosis and appropriate laboratory evaluation for early diagnosis and appropriate treatment.

Case Report: A 10-mo-old boy presented with a rash since 5 mo of age. He first developed vesicular eruptions, associated with irritability, colic, and vomiting but no fever. He had frequent exacerbations and on some occasions associated with severe gastrointestinal upset and difficulty in breathing, rhinorrhea, conjunctivitis, symptoms compatible with anaphylaxis. He recovered on treatment with parenteral dexamethasone and $\mathrm{H} 1$ anti-histamines for few days.

At $8 \mathrm{mo}$ his serum total $\mathrm{IgE}$ level was moderately high $(76 \mathrm{mg} / \mathrm{dl})$ and specific IgE was negative. At 10 mo of age, during hot climate, he had a severe exacerbation and was promptly hospitalized due to extensive hemorrhagic bullae, progressive respiratory distress, vomiting, and fainting. $\mathrm{He}$ was transferred to the pediatric ICU with presumptive diagnosis of septic shock for assisted ventilation, adrenaline IV drip, pulse methylprednisolone $(30 \mathrm{mg} / \mathrm{kg} / \mathrm{day})$, with improvement within 3 days followed by prednisolone $2 \mathrm{mg} / \mathrm{kg} /$ day.

Systemic mastocytosis was suspected. Serum tryptase level was very high at $120 \mathrm{ng} / \mathrm{ml}(\mathrm{Nl}<11)$. Skin biopsy was compatible with bullous mastocytosis. Bone marrow aspirate was normal without significant mast cells, supporting the diagnosis of diffuse cutaneous mastocytosis. C-Kit analysis did not reveal mutation.

Corticosteroids ( $2 \mathrm{mg} / \mathrm{kg} /$ day) and tyrosine kinase inhibitor (imatinib mesylate $340 \mathrm{mg} / \mathrm{m}^{2} / \mathrm{d}$ ) were administered with significant improvement. SCORMA (scoring mastocytosis) decreased from 116 to 5 by the age of $14 \mathrm{mo}$. In addition to an anaphylaxis action plan and avoiding common triggers, the management comprised skin care, tacrolimus ointment, ketotifen, sedating $\mathrm{H} 1$ and $\mathrm{H} 2$-antihistamine. Imatinib was discontinued after $1 \mathrm{mo}$ and prednisolone was gradually withdrawn.

Conclusion: The clinical and laboratory findings in our patient are compatible with diffuse cutaneous bullous mastocytosis. It is the rarest variant of cutaneous mastocytosis and is usually seen from birth to the age of $2 \mathrm{yr}$, with recurrent anaphylaxis, and a guarded prognosis. In addition to avoidance of triggers of mast cell activation and symptomatic therapies, specific therapeutic modalities depend on the severity of the disease, with imatinib reserved for severe cases.

Keywords: anaphylaxis, mastocytosis, bullous rash

P006. Challenges in diagnosing auto-inflammatory disorders in low resource setting

*Tinsae Alemayehu

American Medical Center, Specialty Clinic for Infectious Diseases and Travel Medicine, Addis Ababa, Ethiopia.

Background: With increasing awareness of the PID syndromes and their presentation, more children with primary immune-deficiencies (PID) are being diagnosed in Ethiopia with each passing year.

Methods: The clinical presentation of a young Ethiopian boy suspected of having an auto-inflammatory disorder and the diagnostic and therapeutic challenges in a low income country are outlined.

Results: A three year old Ethiopian boy presented with recurrent respiratory and intestinal infections starting from two years of age. Episodes were notable for subcutaneous nodules shifting positions with each febrile episode, arthralgia, marked leukocytosis and neutrophilia, normal serum immunoglobulin levels and remaining hematologic parameters, normal chest X-ray but mesenteric adenitis, varying levels but persistently elevated CRP even during periods between febrile episodes, negative autoimmune and oncologic markers and negative markers for HIV, TB and other infectious illnesses. He had no atypical post-vaccine reactions and his maternal uncle has an "high predisposition for illnesses". He was circumcised at ten days of age (uneventful) and shed his umbilical cord at one week of age. Febrile episodes are being managed with short-term oral steroids while exploring ways to do genetic testing for autoinflammatory disorders.

Conclusion: There are numerous auto-inflammatory disorders which are increasing in number by the year. Though some members of this group are associated with specific communities - familial Mediterranean fever among Mediterranean populations, hyperimmunoglobulin D syndrome among North European etc, some others like Tumor necrosis factor receptor-associated periodic syndrome can be diagnosed globally. Clinical features of auto-inflammatory disorders overlap and genetic analysis for known mutations is vital for diagnosis. A paucity in genetic labs makes confirmation of such disorders difficult in Africa. But continuing education will help identify common clinical features and avoid unnecessary work-up and antimicrobial treatment for similar patients.

Keywords: Auto-inflammatory disorder, Primary immune-deficiencies, Child, Ethiopia

P007. Disseminated tuberculosis complicating Bacillus CalmetteGuérin (BCG) vaccine as only presentation of Severe combined immunodeficiency (SCID). Report of three cases

*Karmel Omer ${ }^{1}$, Gufran Algaly ${ }^{2}$, Omaima Abdelmajeed Mohamed Salih $^{1,3}$

${ }^{1}$ Tropical disease hospital, Khartoum, Sudan; ${ }^{2}$ Hamad Medical corporation, Doha, Qatar; ${ }^{3}$ Departments of Pediatrics, Faculty of Medicine, Omdurman Islamic University, Khartoum, Sudan

Background: Severe combined immunodeficiency disease (SCID) is a rare primary immunodeficiency disease, usually manifesting in the first 6 month of life with failure to thrive, oral thrush, recurrent respiratory infection and chronic diarrhea. 
Methods: We report a unique presentation of SCID in three male patients. They are an outcome of consanguineous marriage and all received the $\mathrm{BCG}$ vaccine at birth.

Results: All three cases presented with regional lymphadenopathy at age of 3 months that progressed to generalized lymphadenopathy treated with anti-tuberculous treatment. The first and second case were twins. The first had uneventful history until the age of 33 months when he developed multiple suppurative lymph nodes confirmed by biopsy. The second and the third cases were diagnosed with disseminated tuberculosis at age of 24 months as they developed fever, anemia, weight loss, tuberculous peritonitis and lymphadenopathy confirmed by biopsy. After investigations, the first case was diagnosed as CD4, CD16 lymphopenic SCID, the second case as CD4, CD8, CD19, CD16 lymphopenic SCID with hypogammaglobulinemia and the third case as CD3, CD4,CD8 lymphopenic SCID with hypogammaglobulinemia. They received anti-tuberculous treatment, prophylactic Trimethoprim/Sulfamethoxazole and Immunoglobulin infusion. The patients at the time of writing this report are all alive and thriving normally and they did not have any other bacterial, viral or fungal infection, the twins are 3 years old and the third case is 30 month old.

Conclusion: SCID may not exhibit the classical manifestation of recurrent infections, and it may present only as a complication of BCG vaccine alerting to high level of clinical suspicion for such patients especially in Sudan where BCG vaccine is usually given at birth.

Keywords: SCID, Primary immunodeficiency, Disseminated Tuberculosis, BCG vaccine

\section{P008. How the INF-y release assay (IGRA) can suggest a PID}

*Fatima-Ezzohra Eddehbi ${ }^{1}$, Saad Elmoujadili ${ }^{1}$, Imane Brahim ${ }^{1}$, Raja Hazime $^{1}$, Brahim Admou ${ }^{1,2}$

${ }^{1}$ Laboratory of immunology, University Hospital Mohammed VI, Marrakech, Morocco; ${ }^{2}$ Bioscience research laboratory, FMPM, Cadi Ayyad university, Marrakech, Morocco

Purpose: INF-y release assay (IGRA) is primarily used to screen for latent tuberculosis. However, the test may display indeterminate results, which are generally due to a low response to positive control (Mitogen). This can be related to immunodeficiency conditions that negatively impact the production of IFN-y. We sought to demonstrate how an indeterminate IGRA profile can reveal a possible Primary Immunodeficiency Disease (PID).

Methods: The population of this study was selected from 584 cases who initially underwent an IGRA showing 162, 409 and 13 positive, negative and indeterminate cases respectively. After ruling out the other causes of indeterminate IGRA profiles, such as pre-analytical errors $(n=4)$ and acquired immunodeficiency conditions $(n=6), 3$ cases for whom clinical data were in favor of PID benefited from T, B and NK cell phenotyping, using flow cytometry (FacsCanto-II, BD).

Results: The three selected cases were 15 months, 18 months and 25 years old, and all of them had a history of unexplained and recurrent infections. The subpopulation phenotyping results showed: $T$ lymphopenia $(C D 3=980, C D 4=728, C D 8=250 / \mathrm{mm} 3)$, suggesting a cellular deficiency (Case-1); B lymphopenia $(\mathrm{CD} 19=668 / \mathrm{mm} 3)$ in favor of a humoral deficit (Case-2) and NK cell lymphopenia $(C D 16 / 56=10 / \mathrm{mm} 3)$ that may suggest a MSMD (Case-3). Additional investigations are underway for all these cases.

Conclusion: Indeterminate IGRA profile represents a possible PID revealing circumstance. On basis of clinical data, such profile should initiate a rational diagnostic approach including subpopulations phenotyping, immunoglobulin assay, IFN-y/IL12 investigating and others, in order to establish or eliminate a PID.

Keywords: IGRA, indeterminate profile, PID
P009. Neutropenia in patients with sepsis: neutrophil function is the predictor of recovery rather than the count

*Manahil Y Solaiman ${ }^{1}$, Nahla H H Erwa ${ }^{2}$, Osama A Eltayeb ${ }^{3}$, Eman A Fadul $^{3}, *$ Ahmed M Musa ${ }^{1}$

${ }^{1}$ Department of Clinical Pathology Immunology, Institute of Endemic Diseases, University of Khartoum, Sudan; ${ }^{2}$ Department of Microbiology, Faculty of Medicine, University of Khartoum, Sudan; ${ }^{3}$ Flow cytometry laboratory, Khartoum, Sudan.

Background: Sepsis is a clinical syndrome that has physiologic and biochemical abnormalities caused by a dysregulated inflammatory response to infection. It may lead to multiple organ dysfunction syndrome and death. Furthermore, immune diagnostic biomarkers are not available. Neutrophil plays a crucial role in the pathophysiology of sepsis.

Objectives: The study aimed at assessing if there is any correlation between the neutrophils count (ANC) and function (NF) and the stage of sepsis.

Methods: A descriptive cohort hospital-based study was conducted at the ICU at Soba University hospital and Omdurman Military Hospital. Patients were diagnosed using the SIRS defining variables. ANC and NF were measured using whole venous blood samples. Serum samples was separated and stored in $-20^{\circ} \mathrm{C}$. Data were collected at the time of admission and recovery from sepsis.

Results: Eighty patients and 7 healthy controls were enrolled in this study. Patients were categorized into 3 groups; severe sepsis, septic shock and MODS according to standard criteria for definition of sepsis. Seventy patients had septic shock. The mean age was 61.3 \pm 20.1 and male:female ratio was 1.5:1. Seven patients had severe sepsis with a mean age of $46.1 \pm 12.9$ and male to female ratio was $3: 1$. And 3 MODS patients had ANC of $14000 \pm 8040$, and normal NF. Fifty-nine (59/70) patients died and the remaining (11/70) recovered. Those who died showed high mean ANC $(10400 \pm 7360)$ whereas those who recovered showed low mean ANC (6200 $\pm 3010), p=0.001$. The NF showed increment with increasing severity of sepsis, $\mathrm{p}=0.001$.

Conclusions: The neutrophils function could be considered as a predictor of recovery in septic shock, unlike the $\mathrm{ANC}$ which is very variable. One of the study limitations is that G-CSF was not measured.

Keywords: Sepsis, ANC, MODS, NF

Corresponding author: AM Musa, tel: +249918043288 musaam2003@yahoo.co.uk, amusa@iend.org

P010. Variable functional phenotypes of inherited IL-12 receptor variants in patients with Mendelian Susceptibility to Mycobacterial Disease

*Ansia van Coller ${ }^{1}$, Brigitte Glanzmann ${ }^{2,3}$, Marlo Möller ${ }^{2}$, Michael Urban $^{2}$, Craig Kinnear ${ }^{2,3}$, Deepthi Raju Abraham ${ }^{4}$, Monika Esser ${ }^{1,4}$, Richard Glashoff ${ }^{1}$

${ }^{1}$ Immunology Unit, Division of Medical Microbiology, National Health Laboratory Service and Faculty of Medicine and Health Sciences, Stellenbosch University, Tygerberg Hospital, Cape Town, South Africa; ${ }^{2}$ DSI-NRF Centre of Excellence for Biomedical Tuberculosis Research, South African Medical Research Council Centre for Tuberculosis Research, Division of Molecular Biology and Human Genetics, Faculty of Medicine and Health Sciences, Stellenbosch University, Cape Town, South Africa; ${ }^{3}$ South African Medical Research Council Genomics Centre, Cape Town, South Africa; ${ }^{4}$ Paediatric Rheumatology and Immunology Unit, Department of Paediatrics and Child Health, Faculty of Medicine and Health Sciences, Stellenbosch University, Tygerberg Hospital, Cape Town, South Africa 
Purpose: In high TB burden countries such as South Africa, individuals with Mendelian Susceptibility to Mycobacterial Disease (MSMD) are prone to developing severe, persistent, unusual or recurrent (SPUR) TB. Several genes have been associated with MSMD, all resulting in the disruption of the IL-12-IFN- $\gamma$ cytokine axis, which is essential for the effective control of mycobacterial infections. The aim of this study was to assess the immunological phenotypes (relating to the IL-12-IFN- $\gamma$ cytokine pathways) in four patients with likely disease-causing variants identified in the IL-12 receptor genes (IL12RB1 or IL12RB2) and their unaffected family members using a set of in-house functional profiling assays.

Methods: Novel, plausible disease-causing variants were identified in the genes coding for the IL-12 receptor in all four MSMD patients included in the study: IL12RB1 for P1 and P2; P3 had variants in both IL12RBI and IL12RB2; while P4 had a variant identified in IL12RB2. IL-12R $\beta 1$ and IL-12R $\beta 2$ expression was assessed through standard flow cytometric phenotyping and IL-12 receptor signalling was assessed by means of phospho-specific flow cytometry, which detects phosphorylated STAT4 following in vitro stimulation. IL-12-induced IFN- $\gamma$ production was determined by Luminex following 48-hour IL-12 and PHA or BCG costimulation.

Results: The four patients had varying degrees of immune dysfunction, which deviated from the controls as well as from their unaffected family members.

Conclusion: This emphasises the importance of in vitro assessment of genetic variants in patients and their family members to determine functional penetrance of particular gene variants. An accurate molecular diagnosis for MSMD allows for appropriate intervention and treatment for affected individuals.

Keywords: MSMD, Immunodeficiency, TB, IL-12

\section{P011. CD3, CD4 and CD8 in Children with Chronic Diarrhea}

Zeinab A El-Sayed, Maha M Awadalla, *Rasha H El-Owaidy, Lamis M Tawfik, Mohab M Zekry

Pediatric Allergy, Immunology and Rheumatology Unit, Ain Shams University, Cairo, Egypt

Background: Severe defects in T cell number and/or function result in increased susceptibility to severe infections including chronic diarrhea.

Objective: This pilot study aimed at investigating underlying T cell immunodeficiency in Egyptian children with chronic diarrhea through assessment of CD3, CD4 and CD8.

Methods: Thirty children with chronic diarrhea were investigated in comparison to 20 healthy controls. For all subjects, complete blood counts and flow-cytometric analysis of CD3, CD4 and CD8 were done.

Results: Among patients' group, we found five patients with decreased CD3, CD4 and CD8 percentages and counts and CD4/CD8 ratio. In comparison to controls, patients with chronic diarrhea had significantly higher mean white blood cells counts (mean $\left.\pm \mathrm{SD}=13.4 \pm 2.510^{9} / \mathrm{L}\right)$, lower lymphocytic count (mean $\pm \mathrm{SD}=3.8 \pm 1.710^{9} / \mathrm{L}$ ), lower $\mathrm{CD} 4$ and CD8 percentages, lower $\mathrm{CD} 3$ counts (mean $\pm \mathrm{SD}=2.5 \pm 1.210^{9} / \mathrm{L}$ ), lower $\mathrm{CD} 4$ counts (mean $\pm \mathrm{SD}=1.4 \pm 0.710^{9} / \mathrm{L}$ ) and $\mathrm{CD} 8$ counts (mean $\pm \mathrm{SD}=1 \pm 0.510^{9} / \mathrm{L}$ ) and lower $\mathrm{CD} 4 / \mathrm{CD} 8$ ratio. Patients with chronic diarrhea with normal $\mathrm{CD}$ markers were comparable to controls in relation to all studied laboratory parameters. T cell counts and CD4/CD8 ratio correlated negatively with duration of diarrhea and number of previous repeated infections per year and positively with weight percentiles.

Conclusion: Investigating T cell subpopulations is an essential step in the evaluation of patients with chronic diarrhea as this may help reveal an underlying immunodeficiency.

Keywords: Chronic, diarrhea, immunodeficiency, lymphocyte, subsets
P012. Primary Immune Deficiencies in children: follow-up and outcome

*Indou Deme $/ \mathrm{ly}^{1}$, Mame Sokhna Gueye ${ }^{2}$, Awa Kane ${ }^{1}$, Aminata Diop Nakoulima $^{3}$, Ibrahima Diop ${ }^{1}$, Mame Tene Ndiaye ${ }^{1}$, Abou Ba ${ }^{1}$, Idrissa Demba $\mathrm{Ba}^{1}$, Babacar Niang ${ }^{1}$, Aliou Thiongane ${ }^{1}$, YaayJoor Dieng ${ }^{1}$, Yaye Fatou Mbodj/Diop ${ }^{1}$, Ouafae Achnin ${ }^{1}$, Papa Moctar Faye ${ }^{1}$, Amadou Lamine Fall ${ }^{1}$, Ibrahima Diagne ${ }^{4}$, Tandakha Ndiaye Dieye ${ }^{2}$, Ousmane Ndiaye ${ }^{1}$

${ }^{1}$ Albert Royer National Children's Hospital, Cheikh Anta Diop University, Dakar, Senegal; ${ }^{2}$ National Blood Transfusion Center, Cheikh Anta Diop University, Dakar, Senegal; ${ }^{3}$ Dakar Main Hospital (Hôpital Principal), Dakar, Senegal; ${ }^{4}$ Research and Training Unit of Sciences and Health, Gaston Berger University, Saint-Louis, Senegal

Background: Primary Immune Deficiencies (PID) are rare, underdetermined diseases particularly in sub-Saharan Africa. Our aim was to share the results of the follow-up of these patients.

Patients and methods: We conducted a descriptive cross-sectional study at the Albert Royer National Children's Hospital in Dakar, in collaboration with the Immunology laboratory of the National Blood Transfusion Center to include patients with suspected PID, from 2014 to 2021, after ruling out HIV infection. The diagnostic criteria were based on the recommendations of the Moroccan Society for Primary Immunodeficiencies.

Results: Out of 32 patients registered, 16 were included in a follow-up consultation $(50 \%)$. The sex ratio was 0.6 and the mean age at diagnosis was 51.1 months. Consanguinity was observed in half of the patients $(8 / 16)$. The warning signs were mainly infectious (11/16). Eczema and warts were recorded in 3/16 and neurological symptom including ataxia in 3/16. Protein electrophoresis, lymphocyte immunophenotyping and immunoglobulin levels were checked in 10/16, 10/16 and 4/16 children ; respectively. The main PID diagnoses included congenital neutropenia (3/16), SCID (3/16), ataxia telangectasia (3/16), hypogammaglobulinemia $(2 / 16)$, epidermodysplasia verruciformis $(2 / 16)$, Wiskott-Aldrich syndrome $(1 / 16)$, chronic granulomatosis disease (1/16) and Evans syndrome (1/16). The course was marked by relapses-remissions in 6/12 patients and discontinuation of follow-up in 5/16 patients. Bronchiectasis was observed in 2 patients. Mortality was $100 \%$ among SCID and ataxia telangectasia cases.

Conclusion: PIDs are suspected based on atypical clinical signs. Confirmation is difficult in low income countries. The development is marked by a risk of complications or death, hence the need to strengthen clinical-biological collaboration.

Keywords: Primary Immune Deficiencies, Sub-Saharan Africa

P013. First report on the implementation of primary immunodeficiencies (PID) biological diagnosis in Senegal: diagnostic challenges

*Mame Sokhna Guèye ${ }^{1}$, Indou Dème $/ \mathrm{Ly}^{2}$, Awa Kane ${ }^{2}$, Aminata Diop Nakoulima $^{3}$, Diouf MA ${ }^{4}$, Ndour M $^{1}$, Diallo AA ${ }^{1}$, Seck/Diao R ${ }^{5}$, Gadji $\mathrm{M}^{5}$, Babacar Mbengue ${ }^{1}$, Tandakha Ndiaye Dièye ${ }^{5}$

${ }^{1}$ Laboratoire d'Immunologie, Hôpital Aristide le Dantec, Dakar, Senegal; ${ }^{2}$ Albert Royer National Children's Hospital, Cheikh Anta Diop University, Dakar, Senegal; ${ }^{3}$ Dakar Main Hospital (Hôpital Principal), Dakar, Senegal; ${ }^{4}$ Centre hospitalier de Pikine, Dakar, Senegal; ${ }^{5}$ National Blood Transfusion Center, Cheikh Anta Diop University, Dakar, Senegal

Introduction: The primary immunodeficiencies (PID) are a heterogeneous group of more than 330 genetic diseases. They are a large group of diseases affecting the immune system, predisposing the patients to a large spectrum of infections. 
Purpose: To conduct a biological exploration of PID with the available technical means to reinforce an established clinical diagnosis.

Methods: From August 2014 to December 2020, we have retained the suspected cases of PID from several hospital-university structures in Dakar. The included patients were referred to the laboratory of the National Blood Transfusion Centre for blood count and electrophoresis of proteins; and the Immunology service of Dantec hospital for immunophenotyping.

Results: We registered 33 patients, with a sex ratio $(M / F=0.73)$ and an average age of 6.10 years (extremes: 0-42 years). Neutropenia was found in 8 patients, lymphopenia in 6 and thrombocytopenia in 8. Low CD4+, CD8+ and CD19+ count was observed in 8,4 and 8 patients, respectively. Protein electrophoresis showed inflammatory profile in $81.25 \%$ of cases. The immunoglobulin level assay, complement examination and the only one genetic confirmation were done in specialized laboratory. Among other, we obtained 6 cases of syndromic combined immune deficiencies, 4 SCID patients, 4 had humoral deficiency and 2 cases of epidermodysplasia verruciformis.

Conclusion: PIDs are rare diseases but not exceptional. Diagnosis relies on a strong clinical suspicion, with biological orientation, but confirmation is genetic in several cases.

Keywords: primary immunodeficiency, cytometry, electrophoresis

P014. Frequency of Job Buckley syndrome during atopic dermatitis on black skin in the pediatric dermatology department of the Albert Royer Children's Hospital in Dakar

\section{*Mame Tene Ndiaye \\ Albert Royer National Children's Hospital, Cheikh Anta Diop University, Dakar, Senegal}

Purpose: To determine the frequency of Job Buckley syndrome in patients with atopic dermatitis and black skin.

Methods: Prospective observational cross-sectional descriptive study over a 3-month period from April to June 2021. The study population included children seen in consultation at the dermatology department of the Albert Royer Children's Hospital. The diagnosis of atopic dermatitis was made according to the criteria of HANIFIN AND RAJKA. The severity was assessed according to the SCORAD score. Total IgE was measured after taking antihelminthic drugs.

Results: Three cases of Job Buckley syndrome were identified out of 134 cases of atopic dermatitis received during the study period, representing a frequency of $2.24 \%$. The sex ratio was 0.5 . The mean age at diagnosis was 67 months. The mean time to diagnosis was 46 months. The dermatological manifestations were severe atopic dermatitis characterized by extensive pruritic bullous skin lesions with facial involvement, prurigo, and lichenification. Recurrent diffuse bullous impetigo was noted in all cases. The extra-dermatological manifestations included recurrent pneumopathy of ordinary appearance in all cases and recurrent otitis in 1 case. The mean level of total IgE was 3544KUI/l.

Conclusion: The frequency of $2.24 \%$ that we found could be overestimated due to a selection bias linked to the hospital recruitment site. This frequency should be confirmed by a cohort study.

Keywords: Job Buckley syndrome, frequency, atopic dermatitis, black skin

P015. Dermatological manifestations during primary immune deficiency in black skin

*Mame Tene Ndiaye Diop ${ }^{1}$, Niang $\mathrm{MS}^{2}$, Diop A. ${ }^{3}$, Indou Dème Ly ${ }^{1}$, Diop K. ${ }^{4}$, Seck B. Diassé Fall $\mathrm{F}^{1}$, Babacar Niang ${ }^{1}$, Diadié $\mathrm{S}^{4}$, Ndongo $\mathrm{A}^{4}$, Diatta B. $\mathrm{A}^{4}$, Seck B ${ }^{5}$, Deh A ${ }^{4}$, Ndour $\mathrm{N}^{4}$, Ndiaye $\mathrm{C}^{4}$, Sarr M ${ }^{4}$, Diallo $\mathrm{M}^{4}$, Ly $\mathrm{F}^{3}$, Tandakha Ndiaye Dièye ${ }^{2}$, Ousmane Ndiaye ${ }^{1}$, Aziz Bousfiha $\mathrm{AA}^{6}$, Niang $\mathrm{SO}^{4}$

${ }^{1}$ Albert Royer National Children's Hospital, Cheikh Anta Diop
University, Dakar, Senegal; ${ }^{2}$ Service d'immunologie, Université Cheikh Anta Diop, Dakar, Senegal; ${ }^{3}$ Service de Dermatologie, Hôpital Institut d'hygiène sociale, Université Cheikh Anta Diop, Dakar, Senegal; ${ }^{4}$ Hôpital Aristide Le Dantec, Université Cheikh Anta Diop, Dakar, Senegal; ${ }^{5}$ Service de Dermatologie, hôpital Principal de Dakar, Dakar, Senegal; ${ }^{6}$ Service de maladies infectieuses pédiatriques et immunologie clinique, Hôpital d'Enfants Abderrahim Harouchi. CHU Ibn Rochd

Purpose: to describe the dermatological manifestations during primary immune deficiency on black skin.

Methods: Open cohort study of children presenting with dermatological manifestations suspected of primary immune deficiency at the Albert Royer Children's Hospital in Dakar. The diagnosis of primary immune deficiency was based on the phenotypic classification of the International Union of Immunological Societies.

Results: Twenty-eight patients were followed up, 18 girls and 10 boys. The average age was 7 years. The age of onset of symptoms was less than 3 years in 17 cases, between 3 and 10 years in 6 cases, more than 10 years in 5 cases. The dermatological manifestations were: ano-genital condyloma $(n=9)$; Kaposi's disease $(n=2)$, epidermodysplasia verruciformis $(\mathrm{n}=2)$, malignant varicella $(\mathrm{n}=1)$, disseminated tumor molluscum contagiosum $(n=1)$; oral-esophageal candidiasis $(n=1)$; cervicofacial fungal mycetoma $(\mathrm{n}=1)$; Disseminated BCG $(\mathrm{n}=1)$; Scrofuloderma tuberculosis $(n=1)$; Acute lupus ( $n=3)$; Extensive vitiligo $(n=1)$; PAPA syndrome $(n=1)$; Erythroderma $(n=1)$; Acrodermatitis enteropathica $(n=2)$; Cutaneous lymphoma $(n=1)$. Five cases benefited from exploration for immunological typing of the deficiency. This exploration revealed: 1 MHC class II deficiency, 1 hyper Ig E syndrome, 1 primary complement $\mathrm{C} 2$ deficiency and 2 congenital Zinc deficiency.

Conclusion: All cases that benefited from immunological typing were confirmed, suggesting a very high sensitivity of dermatological manifestations for the diagnosis of primary immune deficiencies. This finding should be confirmed by other studies.

Keywords: dermatological manifestations, black skin, primary immune deficiency

P016. Diagnostic spectrum and clinical profile of primary immunodeficiencies in children at an Algerian department of general pediatrics.

*Reda Belbouab ${ }^{1}$, Azzedine Tahiat ${ }^{2}$, S.Hakem ${ }^{1}$, M.Bounaas ${ }^{1}$, Y.Ferhani $^{1}$, S.Sokhal ${ }^{1}$, Kamel Djenouhat ${ }^{2}$, Rachida Boukari ${ }^{1}$

${ }^{1}$ Department of Pediatrics, Mustapha University Hospital, Algiers, Algeria; ${ }^{2}$ Department of Medical Biology, Rouiba Hospital, Algiers, Algeria

Background: Primary immunodeficiencies (PID) are heterogeneous disorders, characterized by variable clinical immunological features. Early diagnosis and appropriate treatment are critical for reducing morbidity and mortality. Purpose: To estimate the disease burden of PID in a department of general pediatrics in Algiers and to appreciate the trends.

Methods: A retrospective single center study conducted in the department of pediatrics at Mustapha Bacha teaching hospital between 2003 and 2020. PID were classified according to the IUIS classification 2019. Results: Eighty-seven PID patients were identified, including 49 boys. Mean age at diagnosis was 32 months. Consanguinity was found in $34 \%$ of cases. Main clinical manifestations were recurrent respiratory infections $(51 \%)$, growth failure (40\%), chronic diarrhea (30\%) and eczema (21\%).Combined T and B cell immunodeficiency (CID) accounted for the majority of cases $(39 \%)$, followed by CID with associated or syndromic features $(23 \%)$, predominantly antibody deficiencies $(17 \%)$, congenital defects of phagocyte $(6,8 \%)$, diseases of immune dysregulation $(6,7 \%)$ and autoinflammatory diseases (5\%). Among CID, SCID was the most common condition (26\%) followed by MHC class II deficiency (8\%). Thirty-one patients had a genetic diagnosis including 8 in Algeria. Bone marrow transplantation (BMT) was performed in 7 patients (3 
Wiskott-Aldrich syndrome, 2 MHC class II deficiency, 1 chronic granulomatous disease and 1 Chediak Higashi) at a mean age of 58 months. The global mortality of our series was $26 \%$.

Conclusion: CID followed by CID with associated or syndromic features were the commonest PID diagnoses in our center. Although access to lymphocyte immunophenotyping has become easy, access to genetic studies and BMT remains less accessible. Long lag time in diagnosis and high mortality in our cohort emphasizes the need for early diagnosis and early referral.

Keywords: Primary immunodeficiencies, clinical manifestations, combined $\mathrm{T}$ and $\mathrm{B}$ cell immunodeficiency

\section{P017. COVID-19 in children: Experience of a children's medical} department

*Reda Belbouab ${ }^{1}$, M. Bounaas ${ }^{1}$, S. Hakem ${ }^{1}$, Y. Ferhani ${ }^{1}$, M. Keddari ${ }^{1}$, M. Noumi $^{1}$, S. Sokhal ${ }^{1}$, F. Fernini ${ }^{1}$, H. Benalioua ${ }^{1}$, N. Benali-khoudja ${ }^{1}$, IS Djillali $^{2}$, N.Ould Rouis ${ }^{2}$, M. Himrane ${ }^{2}$,A. Hadji ${ }^{1}$, H Boudiaf ${ }^{2}$, Rachida Boukari $^{1}$

${ }^{1}$ General pediatric department, ${ }^{2}$ Oncology-pediatric department University hospital Mustapha Pacha - Algiers University, Algiers, Algeria.

Purpose: To investigate the rate of COVID-19 infection in children in University hospital Mustapha Pacha - Algiers.

Methods: This is a retrospective study conducted between April 2020 and June 2021. During this period, 351 children were examined in the pediatric emergency room for suspicion of COVID-19. Demographic, anamnestic (epidemiological contact), clinical, radiological, microbiological data were recorded in the clinical file. The diagnosis of COVID-19 was made based on the following criteria: family contact, compatible symptomatology, positive SARS-CoV-2 serology and / or PCR, chest X-ray or CT.

Results: 351 children aged between 1 month and 15 years with a sex ratio $M$ / F 0.57 were examined. The repartition by age group is as follows: 1 year old 25 (7\%), 1- 5 years 90 (26\%), 6-12 years $128(36 \%), 13-15$ years 108 $(31 \%) .182$ were asymptomatic contact subjects $(52 \%), 169$ were symptomatic $(48 \%)$ of which 26 required hospitalization $(7.4 \%)$. Clinical presentation included general signs (fever, asthenia, headache) $50 \%$, respiratory (cough, runny nose, sore throat) $40 \%$, gastrointestinal signs (diarrhea, vomiting, abdominal pain) $10 \%$. Among the 26 hospitalized children, the findings included pneumonia, MIS-C, gastrointestinal, neurological, severe sepsis, haematological and diabetic ketoacidosis in 12, 5, 3, 2, 2, 1 and 1 cases respectively. Risk factors and comorbidities were identified in 11 children including malignant haematologic disease (6), congenital heart disease (1), chronic liver disease (1), immune deficiency (1), congenital aplastic anemia (1), metabolic disease (cycle disorder urea) (1). Therapies included antibiotics, corticosteroids, anticoagulants and IV immunoglobulins.

Eighteen children (69\%) had a good outcome, 8 (31\%) died (3 with malignant haemopathy, 1 with severe immune deficiency, 1 with severe chronic liver disease, 1 with metabolic disease, 1 with congenital heart disease, 1 with congenital aplastic anemia).

Conclusion: In the majority of cases, the child is asymptomatic or has mild symptoms and the evolution is good.The high number of deaths in this hospital series (case fatality rate of $2.3 \%$ ) is explained by the great vulnerability of children with malignant hemopathy and immunosuppression.

Keywords: Covid-19, comorbidities, intrafamilial contamination

P018. Primary immunodeficiency caused by mutations in RIPK1: report of three cases

*Reda Belbouab, S. Hakem, M. Bounaas, Y. Ferhani, S. Sokhal, Rachida Boukari

Department of Pediatrics, Mustapha University Hospital, Algiers.

Purpose: Mutations in RIPK1 (receptor-interacting serine/threonine kinase 1) were associated with impaired NF-kB activity, defective differentiation of $\mathrm{T}$ and $\mathrm{B}$ cells, increased inflammasome activity, and reduced response to TNFR1-mediated cell death in intestinal epithelial cells. Here we present a case series of 3 cases with confirmed RIPK1 defect.

Results: The series included 3 children; two boys and a girl, of consanguinous parents. Disease onset started very early in life $(6,1$ and 3 months). All had similar clinical presentation with gastrointestinal manifestations suggestive of VEO-IBD with watery diarrhea, abdominal pain, oral aphthous lesions, anal fistulas, growth retardation, ulcerative colitis on endoscopy (A1L2B1 according to Paris classification) and chronic inflammation on histology. Extra-intestinal manifestations included repeated infections, in particular pneumonia and purulent otitis.

The diagnosis was made late at the age of 10,6 and 3 years by molecular biology (D.Kotlarz, University Hospital LMU Munich). Therapeutic management consisted of antibiotics combined with antifungal therapy for the three patients as well as azathioprine treatment in one patient. Progress under treatment led to a reduction in diarrhea and infections.

Conclusion: It is important to identify patients with monogenic Inflammatory Bowel Diseases (IBD) as management may differ from classical IBD. Genetic screening for monogenic IBD is recommended in all patients with very early-onset IBD (6 years), particularly those patients with relevant comorbidity, extraintestinal manifestations, and/or family history. RIPK1 Deficiency causes Immunodeficiency and Intestinal Inflammation.

Keywords: RIPK1, early-onset IBD, monogenic Inflammatory Bowel Diseases

P019. Quantification of Primer Efficiency of the Studies on Severe combined immunodeficiency (SCID) Genotypes Profiles Using realtime PCR Method Based on SYBR Green

*Syarifah Masyitah Habib Dzulkarnain ${ }^{1,2}$, Ilie Fadzilah Hashim ${ }^{1}$, Zarina Thasneem Zainudeen ${ }^{1}$, Intan Juliana Abd Hamid ${ }^{1}$

${ }^{1}$ Primary Immunodeficiency Diseases Group, Cluster of Regenerative Medicine, Advanced Medical and Dental Institute, UniversitiSains Malaysia, Bertam 13200 Kepala Batas, Pulau Pinang, Malaysia; ${ }^{2}$ Faculty of Health Sciences, Universiti Teknologi MARA, Cawangan Pulau Pinang, Bertam Campus 13200 Kepala Batas, Pulau Pinang, Malaysia

Background: The study of the molecular etiologies of severe combined immunodeficiency (SCID) has yielded valuable insights into immune cell development and regulation with the use of gene expression analysis tools that unravel how specific genes respond, thus allow us to infer the consequences on the organism physiology, development and survival. Real-time quantitative PCR (qPCR) is an efficient and frequently used tool to quantify gene expression that enables detection of the PCR product directly during the exponential phase of the reaction in a single step. Two most common detection method used in quantitative gene expression analysis are by using SYBR Green and TaqMan probe. SYBR Green is a non-specific dsDNA-binding dye technically based on binding the fluorescent dye to double-stranded deoxyribonucleic acid (dsDNA) and comparatively less expensive while TaqMan probe is highly specific but more expensive. When using SYBR Green, the most important factor to consider is specificity.

Methods: In this study, we aimed at quantifying primer efficiency of SCID genotypes by using qPCR to determine genetic expression of SCID genotypes profiles with great sensitivity and specificity using SYBR green as a binding dyes for detection.

Results and conclusion: The results using this technique provides a specific and sensitive method to quantify gene expression for a future sequencing-based strategy for the diagnosis of patients with SCID. By using this technique, the use of high performance primer and proper protocols will aid precise detection of gene and avoids the usage of expensive specific probe. 
Keywords: Real-time quantitative PCR (qPCR), Severe combined immunodeficiency (SCID), SYBR Green

Corresponding author: Intan Juliana Abd Hamid; email: intanj@usm.my

Acknowledgement: The authors wish to acknowledge USM for the Short Term Research Grant (USM: 304.CIPPT.6315309) and Ministry of Higher Education Malaysia for sponsoring the student.

P020. Autoimmune cytopenia among 382 patients with inborn errors of immunity

*Azzeddine Tahiat ${ }^{1,2}$, Reda Belbouab ${ }^{2,3}$, Zahir Bouzerar ${ }^{2,4}$, Ouardia Ibsaine $^{2,5}$, Hachemi Maouche ${ }^{2,6}$, Rachida Boukari ${ }^{2,3}$, Kamel Djenouhat ${ }^{1,2}$

${ }^{1}$ Department of Medical Biology, Rouiba Hospital, Algiers, Algeria; ${ }^{2}$ Algiers Faculty of Medicine, University of Algiers, Algiers, Algeria; ${ }^{3}$ Department of Pediatrics, Mustapha Pacha University Hospital; Algiers, Algeria; ${ }^{4}$ Department of Pediatrics, Bab El-Oued University Hospital, Algiers, Algeria; ${ }^{5}$ Department of Pediatrics, Ain Taya Hospital, Algiers, Algeria; ${ }^{6}$ Department of Pediatrics, El-Harrach Hospital, Algiers, Algeria

Background: Inborn errors of immunity (IEIs) are genetic diseases of impaired host immunity. Although recurrent infections comprise the clinical hallmark of IEIs, other noninfectious manifestations such as autoimmune cytopenia (AIC) are often present.

Objective: We aimed to determine the prevalence of AIC in a cohort of Algerian patients with different categories of IEIs.

Methods: In this study, IEI patients were retrospectively screened for the occurrence of AIC. A questionnaire was filled for all patients to evaluate demographic, laboratory, clinical, and molecular data.

Results: A total of 382 IEI patients (213 (56\%) males and 169 (44\%) females), with a median age of 12 years were enrolled in this study. The IEIs were distributed as follows: combined immunodeficiencies (CID; $n=97)$; CID with syndromic features $(n=50)$; predominantly antibody deficiencies (AID; $n=99)$; diseases of immune dysregulation ( $n=24)$; congenital defects of phagocytes $(n=11)$; defects in intrinsic and innate immunity $(n=7)$, complement deficiencies $(n=83)$, other IEIs $(n=11)$. Autoimmune cytopenia was reported in $44(11,5 \%)$ patients, including 29 (7.6\%) with autoimmune hemolytic anemia, $20(5.2 \%)$ with immune thrombocytopenia, $6(1.6 \%)$ with Evans syndrome, and $6(1.6 \%)$ with autoimmune neutropenia. Ten patients $(2.6 \%)$ showed AIC as the first presentation of the disease. AIC was more common in patients whose IEI were classified as diseases of immune dysregulation (33.3\%) and CID (17.5\%). Among CID patients, the highest prevalence of AIC was seen in patients with atypical severe combined immunodeficiency (SCID) (42.1\%). None of the patients with defects of innate immunity and complement deficiencies showed AIC.

Conclusion: Our study confirms the high prevalence of AIC in IEI patients. AIC should be viewed as a warning sign of PID even in the absence of infectious disease. Patients with IEIs, especially those with diseases of immune dysregulation and atypical SCID should be routinely screened for AIC.

Keywords: Autoimmune cytopenia, Inborn errors of immunity, Atypical severe combined immunodeficiency
P021. Autoimmunity in Moroccan patients with common variable immune deficiency *Abire Allaoui ${ }^{1,2}$, Khaoula Mokhantar ${ }^{2}$, Leïla Jeddane ${ }^{2}$, Aziz
Bousfiha $^{2,3}$, Mina Moudatir

${ }^{1}$ Internal Medicine Department, Cheikh Khalifa University Hospital, Mohammed VI University of Health Sciences, Casablanca, Morocco; ${ }^{2}$ Laboratoire d'Immunologie Clinique, Inflammation et Allergie, Faculté de Médecine et de Pharmacie de Casablanca, Hassan II University of Casablanca, Morocco; ${ }^{3}$ Clinical Immunology Unit, Pediatrics Department, El Harouchi University Hospital, Casablanca, Morocco; ${ }^{4}$ Internal Medicine Department, Ibn Rochd University Hospital, Casablanca, Morocco

Purpose: To identify autoimmune features and their frequencies in the moroccan registry of common variable immune deficiency (CVID).

Methods: It is a cross-sectional study that was conducted in July 2021 in Morocco. We have used the moroccan registry of CVID to collect demographic, clinical and biological data of the patients. We have compared between two groups, one group with autoimmune manifestations and the other group was free from any autoimmune or inflammatory involvement. For data analysis, we have used SPSS 25 software, $P$ value was considered significant when $p \leq 0.05$. The patients consent was obtained.

Results: Twenty-six patients were diagnosed with CVID. Mean age of diagnosis was at 25,38 $\pm 15,4$, and mean age of onset was at 18,8 $\pm 15,3$. Sex ratio was 0,73 . Consanguinity was present in $46,5 \%$ of the cases. The revealing sign was sinopulmonary involvement in $61,5 \%$ of the patients, followed by diarrhea $(7,7 \%)$ and autoimmune manifestations $(7,7 \%)$. During the disease course, half of our patients presented with autoimmune manifestations, lymphoproliferation was a complication in $42,3 \%$ of the cases. The main autoimmune features were: immune thrombocytopenic purpura, autoimmune hemolytic anemia, vitiligo, granulomatosis, arthritis, and lupus. Two patients presented with coeliac-like disease, three other patients had a Crohn-like disease, and four more patients were diagnosed as having sarcoidosis-like manifestations.

Autoimmune complications were prevalent in women without a statistic significance. Patients with no consanguinity were having more autoimmune manifestions, $p$ value was non significant. Lymphoprolifrative disease was associated with autoimmunity $(\mathrm{p}=0,005)$. Arthritis was prevalent in patients with autoimmunity $(\mathrm{p}=0,01)$.

Immunoglobuline therapy was used in $92,3 \%$ of patients, corticosteroids were added in $42,3 \%$ of cases along with immunosuppressors in $11,5 \%$. Disease course was good in $69,2 \%$.

Conclusion: Autoimmunity is frequent in patient with CVID, especially autoimmune cytopenia. It should be searched for because the treatment depends on it, to enhance life quality of patients and to avoid severe and lethal complications.

Keywords: Autoimmunity, common variable immune deficiency, primary immune deficiency

Publisher's Note Springer Nature remains neutral with regard to jurisdictional claims in published maps and institutional affiliations. 